\title{
Model-based iterative reconstruction in pediatric chest CT: assessment of image quality in a prospective study of children with cystic fibrosis
}

\author{
Frédéric A. Miéville • Laureline Berteloot • Albane Grandjean • \\ Paul Ayestaran • François Gudinchet • Sabine Schmidt • \\ Francis Brunelle • François O. Bochud • Francis R. Verdun
}

Received: 26 March 2012 /Revised: 8 October 2012 / Accepted: 16 October 2012 /Published online: 7 December 2012

(C) Springer-Verlag Berlin Heidelberg 2012

\begin{abstract}
Background The potential effects of ionizing radiation are of particular concern in children. The model-based iterative reconstruction $\mathrm{VEO}^{\mathrm{TM}}$ is a technique commercialized to improve image quality and reduce noise compared with the filtered back-projection (FBP) method.

Objective To evaluate the potential of $\mathrm{VEO}^{\mathrm{TM}}$ on diagnostic image quality and dose reduction in pediatric chest $\mathrm{CT}$ examinations.

Materials and methods Twenty children (mean 11.4 years) with cystic fibrosis underwent either a standard CT or a moderately reduced-dose CT plus a minimum-dose CT performed at $100 \mathrm{kVp}$. Reduced-dose CT examinations consisted of two consecutive acquisitions: one moderately reduced-dose $\mathrm{CT}$ with increased noise index $(\mathrm{NI}=70)$ and one minimum-dose CT at CTDI ${ }_{\mathrm{vol}} 0.14 \mathrm{mGy}$. Standard CTs were reconstructed using the FBP method while low-dose CTs were reconstructed using FBP and VEO. Two senior
\end{abstract}

F. A. Miéville $(\bowtie) \cdot$ F. O. Bochud $\cdot$ F. R. Verdun Institute of Radiation Physics, Lausanne University Hospital,

Grand-Pré 1,

1007 Lausanne, Switzerland

e-mail: frederic.mieville@gmail.com

L. Berteloot $\cdot$ F. Brunelle

Department of Pediatric Radiology,

Necker Children's Hospital of Paris and University of Paris Descartes,

Paris, France

\section{A. Grandjean · P. Ayestaran}

General Electric Medical Systems Europe,

Paris, France

F. Gudinchet $\cdot$ S. Schmidt

Department of Radiology, Lausanne University Hospital,

Lausanne, Switzerland radiologists evaluated diagnostic image quality independently by scoring anatomical structures using a four-point scale $(1=$ excellent, $2=$ clear, $3=$ diminished, $4=$ non-diagnostic $)$. Standard deviation (SD) and signal-to-noise ratio (SNR) were also computed.

Results At moderately reduced doses, VEO images had significantly lower SD $(P<0.001)$ and higher SNR $(P<0.05)$ in comparison to filtered back-projection images. Further improvements were obtained at minimum-dose CT. The best diagnostic image quality was obtained with VEO at minimumdose $\mathrm{CT}$ for the small structures (subpleural vessels and lung fissures) $(P<0.001)$. The potential for dose reduction was dependent on the diagnostic task because of the modification of the image texture produced by this reconstruction.

Conclusions At minimum-dose CT, VEO enables important dose reduction depending on the clinical indication and makes visible certain small structures that were not perceptible with filtered back-projection.

Keywords CT · VEO - Children · Iterative reconstruction · Chest examination

\section{Introduction}

CT scanning has emerged as the gold standard imaging modality because of its high sensitivity for detecting small structures with respect to chest radiography. This feature has led to a dramatic increase of CT examinations in the population and gives rise to particular concern because of the potential negative effects of ionizing radiation [1]. During the last 25 years, the number of CT examinations has increased from 3 million to more than 72 million in the United States [2]. A similar trend has been observed in children, leading to more than 4 million 
CT examinations in 2001 and even more today [1, 2]. Because children are more sensitive to ionizing radiation and have an extended lifespan in comparison to adults, dose reduction should be considered the first priority and every possible effort to reduce children's exposure to radiation should be undertaken to decrease cancer risk attributed to CT [3, 4].

During the last few years, CT scanner manufacturers have developed strategies to reduce patient exposure without diminishing the diagnostic quality of examinations [5-7]. Among recent techniques, iterative reconstruction methods tailor-made for CT have been playing a major role. Since 2009, several studies have demonstrated dose reductions from $18 \%$ up to $65 \%$ using iterative techniques, depending on diagnostic and patient requirements [8-20], and a possible dose reduction of $36 \%$ was estimated for pediatric cardiac CT examinations [21]. Although these techniques have been designed mainly to reduce image noise, GE Healthcare has recently launched a new algorithm, the first model-based iterative reconstruction, called $\mathrm{VEO}^{\mathrm{TM}}$ (GE Healthcare, Milwaukee, WI) [22]. Unlike its first iterative reconstruction, the adaptive statistical iterative reconstruction, VEO further reduces image noise and improves spatial resolution by taking into account the optics system of the CT. VEO appears particularly well-adapted to children with chronic diseases, such as cystic fibrosis (CF), who require lifelong follow-up examinations and are exposed to potentially high cumulative radiation doses.

VEO has recently been studied using physical metrics computed on phantom images [23]. However, to the best of our knowledge, no study has been performed to determine the potential of VEO to improve the diagnostic image quality and to reduce radiation dose in clinical use for children. Thus, for most standard examinations such investigations are necessary before the technique can be fully accepted as routine.

The purpose of this study was to evaluate whether chest CT examinations performed on children and young adults using reduced tube voltages and doses could produce images acceptable from a diagnostic point of view when reconstructed with VEO. Two radiologists from two medical centers evaluated the visibility of diagnostic criteria from CT chest images of children scanned using standard, moderately reduced-dose and minimum-dose protocols. The diagnostic image quality as well as objective measurements like CT number, image noise and signal-to-noise ratio (SNR) obtained from images reconstructed with VEO were compared with those obtained from images reconstructed with filtered back-projection (FBP).

\section{Materials and methods}

Iterative reconstruction method: $\mathrm{VEO}^{\mathrm{TM}}$

$\mathrm{VEO}^{\mathrm{TM}}$ is a model-based iterative reconstruction technique with the potential to greatly reduce image noise and improve spatial resolution, conspicuity and overall image quality with respect to the traditional FBP method [22]. While adaptive statistical iterative reconstruction takes into account the data statistics using a Poisson-Gaussian distribution model and a nonlinear cost function to adaptively reduce noise in homogeneous regions and preserve high-resolution detail, VEO additionally includes an improved description of the CT system optics. Voxel volumes of the scanned object, focal spot size and the active area size of the detector are taken into consideration. Moreover, to enhance model precision of the CT scanner, complex mathematical formulations were determined to account for physical effects such as beam hardening, scatter and metal attenuation artifacts. However, the cost of VEO complexity is a longer computation time than FBP. Today, several minutes are required to reconstruct $\mathrm{CT}$ images using the VEO technique. Moreover, like the first iterative reconstructions, VEO images have an unusual appearance in comparison to FBP images that could require adaptation time for radiologists [8, 9]. To understand the impact of this modification of image appearance, it is crucial to evaluate image quality not only in terms of objective measurements such as image noise but also based on a diagnostic objective.

\section{Study population}

Twenty children (15 boys and 5 girls) with CF lung disease requiring a follow-up CT were included in this study. From May to November 2011, children were randomly selected to have either a standard examination (group A) or a moderately reduced-dose CT followed by a minimum-dose CT examination (group B). Children were considered ineligible if they were outside the 7- to 18-year range in age or were unable to hold their breath for the duration of the acquisition. Information about thorax morphology (height and width) was measured at the heart level from scout acquisitions. An equivalent diameter $\left(D_{\mathrm{eq}}\right)$ was computed to account for the child's morphology as proposed by Boone et al. [24]. Patient-equivalent diameter was defined as the diameter of a circle that had the same area as the one of an ellipse that has its minor and major axes equal to the thorax height and width of the child.

Institutional and ethical review boards of the participating hospitals approved the study protocol. While children in group A underwent one CT acquisition, children in group B underwent two consecutive acquisitions (a moderately reduced-dose acquisition followed by a minimum-dose acquisition) but for a cumulative dose smaller or equal to the one they would have received had they been in group A. Parents of children in both groups were systemically 
informed and a written consent was obtained before each $\mathrm{CT}$ examination. All the $\mathrm{CT}$ raw data and $\mathrm{CT}$ data files were rendered anonymous.

\section{CT scanning protocols}

Acquisitions were performed on a 64-MDCT scanner (Discovery 750HD; GE Healthcare, Milwaukee, WI) with the child in the supine position and without intravenous contrast media injection. All children received similar instructions so the image acquisition could be performed in inspiratory breath-hold. The five protocols used in this study are shown in Table 1. For all examinations, the tube voltage was fixed at $100 \mathrm{kVp}$. To take into account each child's morphology, standard-dose FBP, moderately reduced-dose FBP and moderately reduced-dose VEO protocols were adapted to each child's body using the automatic current modulation system through the noise index (NI) and fixed current boundaries. Moderately reduced-dose FBP and moderately reduceddose VEO protocols were developed step-by-step over a period of a month (May 2011) by empirically increasing the NI and reducing the tube current limits to ensure a diagnostic image quality for moderately reduced-dose FBP chest examinations. After this adjusting period, these protocols were used to scan children from group B. On the other hand, minimum-dose protocols (FBP and VEO) were established without the automatic current modulation system. To deliver the smallest possible dose at $100 \mathrm{kVp}$, we used the minimum values that can be set at the CT console unit, namely a tube current of $10 \mathrm{~mA}$, a rotation speed of $0.4 \mathrm{~s} /$ rot and a pitch of 1.375 .

Table 1 CT scanning parameters and reconstruction algorithms of the five protocols used in this study. STD Standard (standard-dose) with $F B P$ reconstruction, $L D$ low dose (moderately reduced dose) with $F B P$
Measurement of radiation dose

The measurement of the volume CT dose index $\left(\mathrm{CTDI}_{\mathrm{vol}}\right)$ was performed in a CTDI phantom of diameter $32 \mathrm{~cm}$ with a 10-cm long CT pencil ion chamber connected to an electrometer (Radcal 1035-10.3 CTDI chamber, MDH 1015 electrometer; Radcal, Monrovia, CA) to verify the accuracy of the CTDI ${ }_{\mathrm{vol}}$ displayed at the CT unit. The ion chamber and electrometer were calibrated in RQR9 and RQA9 beams according to IEC 61267 [25] and traceable to the National Physical Laboratory (Teddington, UK). The $\mathrm{CTDI}_{\mathrm{vol}}$ was calculated by dividing the weighted CTDI $\left(\mathrm{CTDI}_{\mathrm{w}}\right)$ by the pitch value, according to its definition [26, 27].

Image reconstruction

A dedicated CT console unit (GE Healthcare, Milwaukee, WI) designed for VEO image reconstructions was used to reconstruct the raw data from the patient examinations. Contrary to the FBP method and adaptive statistical iterative reconstruction, only the standard reconstruction kernel was available with VEO. Axial images were first reconstructed in the axial plane, with a display field of view adapted to the child's morphology (range: $235-414 \mathrm{~mm}$ ) and a matrix of $512 \times 512$ pixels. Two slice thicknesses were obtained: $0.625-\mathrm{mm}$ (native slices) and $1.25-\mathrm{mm}$ axial slices. Then, using the reformat software available on an Advantage Workstation (GE Healthcare, Milwaukee, WI), native $0.625-\mathrm{mm}$ axial images were reconstructed in the coronal plane based on maximum-intensity projections (MIP) to produce contiguous coronal images of 1.41-mm slice thickness.

and $V E O$ reconstructions and $U L D$ ultra low dose (minimum dose) with $F B P$ and $V E O$ reconstruction

\begin{tabular}{|c|c|c|c|c|c|}
\hline \multicolumn{2}{|l|}{ Standard dose } & \multicolumn{2}{|c|}{ Moderately reduced dose } & \multicolumn{2}{|l|}{ Minimum dose } \\
\hline Protocol & STD FBP & LD FBP & LD VEO & ULD FBP & ULD VEO \\
\hline Detector config. (rows x mm) & $64 \times 0.625$ & $64 \times 0.625$ & & $64 \times 0.625$ & \\
\hline Voltage $(\mathrm{kVp})$ & 100 & 100 & & 100 & \\
\hline Scan field of view & Medium body & Medium body & & Medium body & \\
\hline Tube current (mA) & $50-300$ & $30-120$ & & 10 & \\
\hline Noise index (HU) & 32 & 70 & & N/A & \\
\hline Gantry rotation time (s) & 0.4 & 0.4 & & 0.4 & \\
\hline Tube current time (mAs) & Variable & Variable & & 4 & \\
\hline Acquisition mode & Helical & Helical & & Helical & \\
\hline Pitch & 1.375 & 1.375 & & 1.375 & \\
\hline Reconstruction algorithm & FBP & FBP & VEO & FBP & VEO \\
\hline Reconstruction filter & Standard & Standard & & Standard & \\
\hline Display field of view (mm) & Variable & Variable & & Variable & \\
\hline Axial slice thickness (mm) & 1.25 & 1.25 & & 1.25 & \\
\hline Coronal slice thickness (mm) & 1.41 & 1.41 & & 1.41 & \\
\hline
\end{tabular}


Clinical image quality of FBP and VEO examinations

Diagnostic image quality was assessed using a four-point rating scale $(1=$ excellent, $2=$ clear, $3=$ diminished, 4 = non-diagnostic) on a predefined set of anatomical structures relevant for chest examinations. Two board-certified radiologists (both with more than 20 years of experience) working in two different large hospitals established a set of anatomical structures to assess the diagnostic image quality of the CT chest examinations. In addition to this set of structures, the subjective image noise was evaluated in order to represent the overall subjective image quality of the examinations. The list of criteria proposed to score image quality was accepted in consensus by both radiologists. Table 2 presents the six criteria (five chest structures and subjective image noise) retained as relevant to assess the quality of chest examinations.

Prior to the radiologists' scoring, clinical data from the children as well as information about acquisition and reconstruction were removed. Then the examinations were separated into two sets: the axial image set and the coronal image set, and each set was independently randomized for both reconstructions (FBP and VEO).

For each randomized set, the two radiologists independently scored image quality with the following 4-point scale: an excellent visibility of the anatomical structure with distinct anatomical detail that could be expected to be superior to the standard-dose CT was rated with a score of 1 (excellent image quality); an image with clear structure visibility and details that could be considered equivalent to standard-dose CT was rated with a score of 2 (image quality good for diagnostic requirements); a suboptimal structure visibility with inaccurate details and an overall aspect lower than what could be expected at standard-dose CT was given a score of 3 (image quality tolerable but diminished for diagnostic requirements); finally, an unacceptable structure visibility that could impact the diagnostic accuracy was given a score of 4 (image quality inadequate for diagnostic requirements). To minimize bias in scoring, the window/level setting was standardized to 1,400 / 500 (lung settings). After a training period was performed the

Table 2 Set of criteria established to assess the diagnostic image quality of chest follow-up examinations

\begin{tabular}{ll}
\hline Diagnostic criteria & \\
\hline Global criteria & Subjective image noise \\
Normal anatomical structures & Central bronchi \\
& Lung vessels \\
& Peripheral bronchi wall thickness \\
& Subpleural vessels \\
& Lung fissure \\
\hline
\end{tabular}

two observers carried out their analyses independently and separately in a quiet room with low-ambient lighting on the same workstation equipped with a diagnostic screen (RadiForce MX210; Eizo, Ishikawa, Japan). In total, each radiologist scored 600 structures $(10$ patients $\times 5$ protocols $\times 6$ structures $\times 2$ reconstruction planes).

Objective image-quality measurements

CT numbers and objective image noise (the pixel standard deviation) were measured for the children's examinations in both groups. A region of interest (ROI) approximately $80 \mathrm{~mm}^{2}$ was placed in the descending chest aorta at the child's heart level. Previous metrics were used to compute SNR, defined as the CT number divided by SD. Objective measurement values were expressed as mean \pm standard error of the mean [28].

Statistical analysis

All statistical analyses were performed using the commercial SPSS software (version 15.0; SPSS, Chicago, IL). Child's age and morphology as well as objective image quality were investigated using an independent sample $t$-test while radiologist score values were analyzed by using a repeated-measures analysis of variance (ANOVA) procedure (normality assumption obtained with the ShapiroWilk W test), with a Bonferroni post hoc comparison to control for multiple pair-wise comparisons. Statistical significance was accepted at the $95 \%$ confidence level $(P$ value $<0.05)$ for all repeated measures and analyses. Rating values were expressed as mean \pm standard error of the mean (normal distribution assumption).

\section{Results}

The analysis performed on children in groups A and B showed no significant differences in age $(t$-test, $P=0.8)$ or $D_{\text {eq }}(t$-test, $P=0.5)$ as shown in Table 3 .

Table 3 Patient characteristics in groups A and B. Data are mean \pm standard error of the mean. $D_{e q}$ Equivalent diameter

\begin{tabular}{lcc}
\hline Characteristic & Standard dose & $\begin{array}{l}\text { Moderately reduced } \\
\text { dose }+ \text { minimum dose } \\
\text { Group B }\end{array}$ \\
\hline Patients & 10 & 10 \\
Boys & 8 & 7 \\
Girls & 2 & 3 \\
Mean age (years) & $11.2 \pm 1.2$ & $11.6 \pm 1.2$ \\
Mean $D_{\text {eq }}(\mathrm{cm})$ & $21.0 \pm 0.9$ & $21.7 \pm 0.9$ \\
\hline
\end{tabular}




\section{Radiation doses}

For group A, which was scanned with our standard FBP protocol, the average $\mathrm{CTDI}_{\mathrm{vol}}$ and average DLP were $1.84 \mathrm{mGy}$ (range: $0.86-3.28 \mathrm{mGy}$ ) and $63.5 \mathrm{mGy} \cdot \mathrm{cm}$ (range: $25.0-133.6 \mathrm{mGy} \cdot \mathrm{cm}$ ). These values are below the diagnostic reference level $\left(\mathrm{CTDI}_{\mathrm{vol}}\right.$ and $\left.\mathrm{DRL}\right)$ recommended for pediatric chest CT examinations by the Swiss Federal Office of Public Health $\left(\mathrm{CTDI}_{\mathrm{vol}}\right.$ and DLP for chest CT are $4.3 \mathrm{mGy}$ and $6.8 \mathrm{mGy}$ as well as $105 \mathrm{mGy} \cdot \mathrm{cm}$ and $205 \mathrm{mGy} \cdot \mathrm{cm}$ for 6 - to 10 -year-old children and 11- to 15-year-old children, respectively) [29]. For group $\mathrm{B}$, the average $\mathrm{CTDI}_{\mathrm{vol}}$ and average DLP were $0.78 \mathrm{mGy}$ (range: $0.43-1.37 \mathrm{mGy}$ ) and $25.7 \mathrm{mGy} \cdot \mathrm{cm}$ (range: $11.6-56.1 \mathrm{mGy} \cdot \mathrm{cm}$ ) for the moderately reduced-dose protocols while $0.14 \mathrm{mGy}$ and $4.57 \mathrm{mGy} \cdot \mathrm{cm}$ (range: 3.73-6.04 $\mathrm{mGy} \cdot \mathrm{cm}$ ) were obtained with the minimum-dose protocols. The resulting cumulated average DLP for group B was $30.3 \mathrm{mGy} \cdot \mathrm{cm}$ and was approximately half the mean DLP value obtained for group A.

According to the study by Deak et al. [30], who computed conversion factors from DLP to effective dose using Monte Carlo simulations, the effective dose for a 10-yearold undergoing one of our $100 \mathrm{kVp}$-based chest examinations (coefficient factor, $0.0235 \mathrm{mSv} \mathrm{mGy}^{-1} \mathrm{~cm}^{-1}$ ) would be $1.6 \mathrm{mSv}, 0.60 \mathrm{mSv}$ and $0.11 \mathrm{mSv}$ for the standard, moderately reduced-dose and minimum-dose acquisitions, respectively. This corresponds to an effective dose reduction of $60 \%$ for the moderately reduced-dose protocols and $92 \%$ for the minimum-dose protocols. Note that with the latter protocol, the radiation dose is roughly twice that delivered for a chest radiograph ( $0.05 \mathrm{mSv}$ for posteroanterior chest) [31].

Objective image-quality analysis

Table 4 summarizes the objective image quality obtained using metric measurements. In the axial plane, the objective image noise (SD) is increased by $38 \%$ $(40 \pm 3$ Housfield unit [HU] vs $25 \pm 2 \mathrm{HU} ; P<0.001)$ between moderately reduced-dose FBP and standard FBP images, while SD is decreased by $44 \%$ $(14 \pm 1 \mathrm{HU}$ vs $25 \pm 2 \mathrm{HU} ; P<0.001)$ between moderately reduced-dose VEO and standard FBP images. Further noise reduction, $65 \%(14 \pm 1 \mathrm{HU}$ vs $40 \pm 3 \mathrm{HU}$; $P<0.001)$, is even reached when comparing moderately reduced-dose VEO and moderately reduced-dose FBP images. For minimum-dose protocols, image noise decreased by $77 \%(19 \pm 2 \mathrm{HU}$ vs $81 \pm 6 \mathrm{HU} ; P<0.001)$ between VEO and FBP images. Similar trends were observed for the coronal plane. Relative noise reductions of $55 \%(14 \pm 1 \mathrm{HU}$ vs $31 \pm 2 \mathrm{HU} ; P<0.001)$ and $75 \%(21 \pm 1 \mathrm{HU}$ vs $84 \pm 7 \mathrm{HU} ; P<0.001)$ are obtained between moderately reduced-dose VEO and moderately reduced-dose FBP protocols and between minimum-dose VEO and minimum-dose FBP protocols, respectively.

Because no significant difference in the mean CT numbers was observed with doses and reconstruction techniques $(P>0.28)$, SNR values are dependent mainly on SD measurements. In the axial plane, SNR with VEO is increased by factors of $3.0(3.3 \pm 0.6$ vs $1.1 \pm 0.2 ; P<0.05)$ and $3.3(2.0 \pm 0.3$ vs $0.6 \pm 0.1$; $P<0.001)$ in comparison with FBP when acquisitions are performed with moderately reduced dose and minimum dose, respectively, while in the coronal plane, the associated factors are $2.4(1.4 \pm 0.2$ vs $3.3 \pm 0.3 ; P<0.001)$ and $2.7(0.7 \pm 0.1$ vs $2.4 \pm 0.2 ; P<0.001)$.

Diagnostic image-quality assessments

Figures 1 and 2 show axial and coronal chest CT images obtained with the five pediatric protocols investigated in this study. Diagnostic image quality results are represented in Figs. 3, 4 and 5. A statistically significant difference between VEO and FBP protocols

Table 4 Assessment of objective image quality when measured in the descending thoracic aorta on patient images. Data are mean \pm standard error of the mean

\begin{tabular}{|c|c|c|c|c|c|c|}
\hline & \multirow[t]{2}{*}{ Metric } & \multicolumn{5}{|l|}{ Protocols } \\
\hline & & Standard FBP & $\begin{array}{l}\text { Moderately } \\
\text { reduced-dose FBP }\end{array}$ & $\begin{array}{l}\text { Moderately } \\
\text { reduced-dose VEO }\end{array}$ & Minimum-dose FBP & Minimum-dose VEO \\
\hline \multirow[t]{3}{*}{ Axial } & Mean CT number (HU) & $39 \pm 4$ & $42 \pm 6$ & $43 \pm 5$ & $42 \pm 4$ & $35 \pm 4$ \\
\hline & Mean image noise (HU) & $25 \pm 2$ & $40 \pm 3$ & $14 \pm 1$ & $81 \pm 6$ & $19 \pm 2$ \\
\hline & Mean signal-to-noise ratio (-) & $1.6 \pm 0.3$ & $1.1 \pm 0.2$ & $3.3 \pm 0.6$ & $0.6 \pm 0.1$ & $2.0 \pm 0.3$ \\
\hline \multirow[t]{3}{*}{ Coronal } & Mean CT number (HU) & $42 \pm 4$ & $46 \pm 3$ & $43 \pm 3$ & $54 \pm 2$ & $51 \pm 4$ \\
\hline & Mean image noise (HU) & $21 \pm 2$ & $31 \pm 2$ & $14 \pm 1$ & $84 \pm 7$ & $21 \pm 1$ \\
\hline & Mean signal-to-noise ratio (-) & $2.2 \pm 0.3$ & $1.4 \pm 0.2$ & $3.3 \pm 0.3$ & $0.7 \pm 0.1$ & $2.4 \pm 0.2$ \\
\hline
\end{tabular}


Fig. 1 At reduced dose and minimum dose, the conspicuity of large structures like the central bronchi is slightly higher with VEO while for small structures, like the subpleural vessels and lung fissures, the visibility was higher with VEO in comparison to filtered backprojection (FBP). a Axial chest $\mathrm{CT}$ in a 10 -year-old boy included in group A (standard protocol: CTDIvol $=$ 1.99 mGy). b-e Axial chest CT in an 8-year-old boy included in group B (reduced dose protocol: CTDIvol $=0.73 \mathrm{mGy}$; minimum dose protocol: CTDIvol $=0.14 \mathrm{mGy}$ )

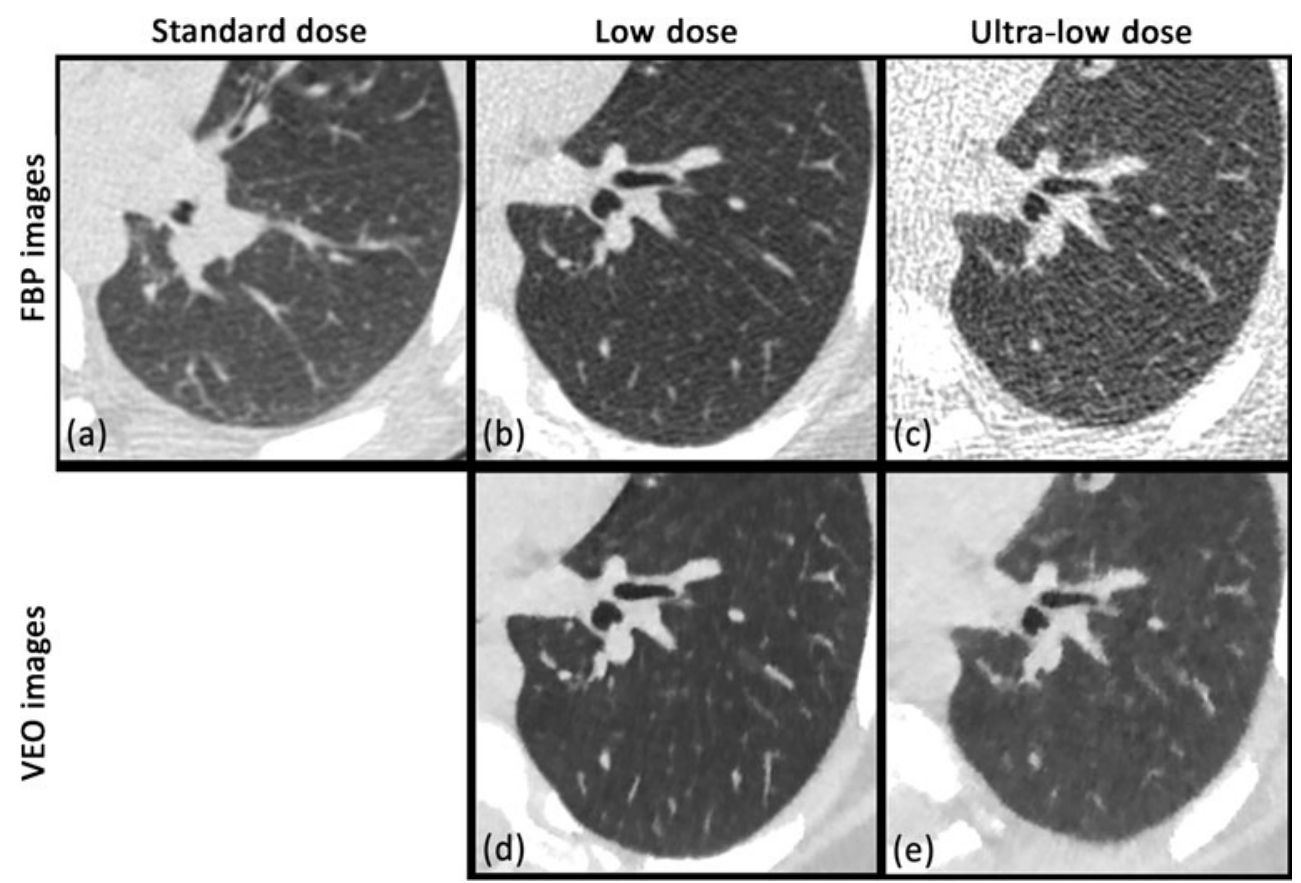

was obtained for axial and coronal images (repeated measures ANOVA, $P<0.05$, for all pair-wise comparisons) while no statistically significant difference within protocols was found between radiologists (repeated measures ANOVA, $P>0.165$, for all pair-wise comparisons).

For axial and coronal images, the two radiologists ranked subjective image noise of moderately reduceddose VEO and minimum-dose VEO images as reduced or identical compared with that of standard FBP images. For the axial plane, subjective image noise was decreased by $54 \%$ (mean scores 1.1 vs 2.4; repeated measures ANOVA, $P<0.001$, for all pair-wise comparisons) with moderately reduced-dose VEO and by $49 \%$ (mean scores 1.8 vs 3.5; repeated measures ANOVA, $P<0.001$, for all pair-wise comparisons) with minimumdose VEO in comparison to moderately reduced-dose FBP and minimum-dose FBP, respectively, while the
Fig. 2 At reduced dose and minimum dose, the conspicuity of large structures like the central bronchi was higher with VEO. For small structures, like the subpleural vessels and lung fissures, the visibility was strongly enhanced, thus higher with VEO in comparison to FBP, where some structures were not discernable. a Coronal chest $\mathrm{CT}$ in a 10 -year-old boy included in group A. b-e Coronal chest CT in an 8-year-old boy included in group B (same patients and acquisition as in Fig. 1)

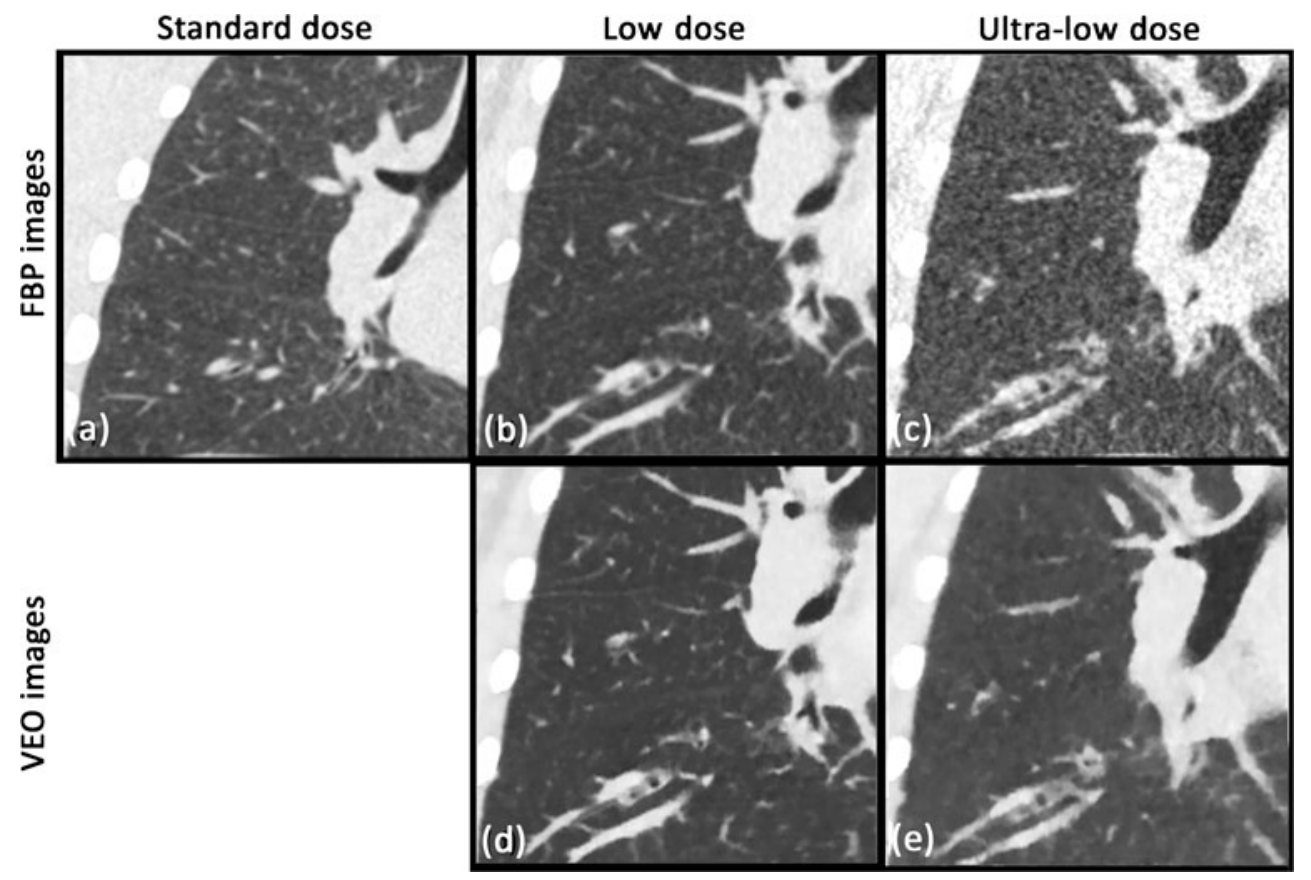




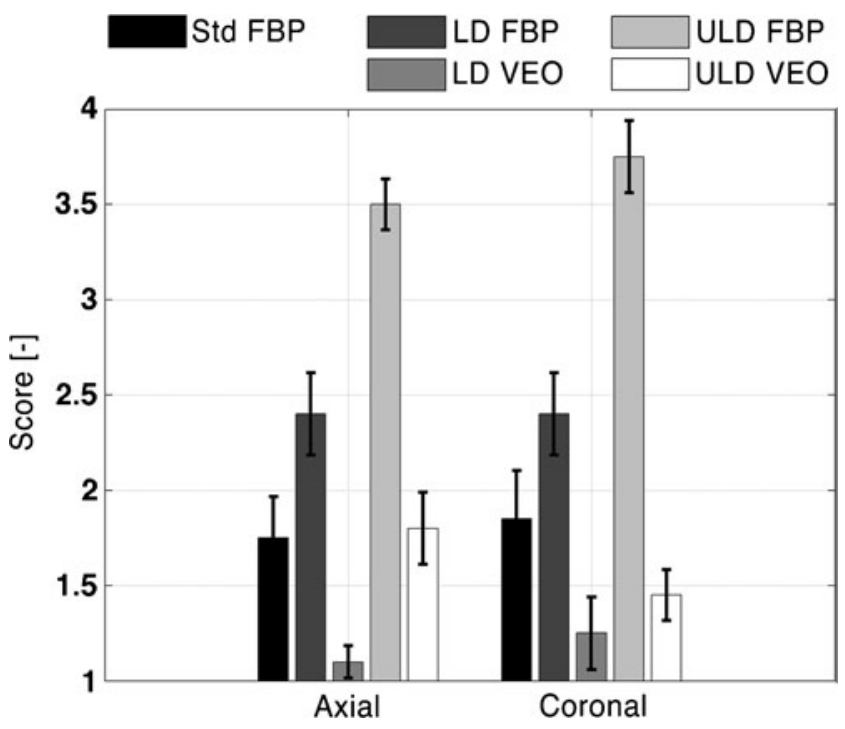

Fig. 3 Overall subjective noise (radiologist scores) obtained from examinations reconstructed in the axial and coronal planes. With VEO, the structure visibility appears to be higher in the coronal plane than in the axial plane, especially for small structures $(P<0.001)$

corresponding decreases were $46 \%$ (mean scores 1.3 vs 2.4; repeated measures ANOVA, $P<0.001$, for all pair-wise comparisons) and $61 \%$ (mean scores 1.5 vs 3.8; repeated measures ANOVA, $P<0.001$, for all pairwise comparisons) for the coronal plane as shown in Fig. 3.

Figures 4 and 5 show the diagnostic image quality of the anatomical structures when visualized in the axial and coronal planes, respectively. For both planes, the large structures (central bronchi and lung vessels) seem to behave differently from the small structures (periphery bronchi wall thickness, subpleural vessels and lung fissures). In the axial plane, the visibility of the central bronchi and lung vessels was not significantly different among standard FBP, moderately reduced-dose FBP and moderately reduced-dose VEO in spite of the important dose reduction (repeated measures ANOVA, $P>0.517$, for all pair-wise comparisons). The same results were obtained for small structures between moderately reduced-dose VEO and moderately reduced-dose FBP protocols (small structures mean scores $1.6 \mathrm{vs} 2.0$; repeated measures ANOVA, $P=0.06$, for all pair-wise comparisons). Nevertheless, both scores were associated with "excellent" or "clear" structure visibility.

In the coronal plane, no significant differences were observed with moderately reduced-dose VEO and moderately reduced-dose FBP for the small and large structures (repeated measures ANOVA, $P>0.05$, for all pair-wise comparisons) and the associated scores led to an "excellent" or "clear" structure.

For the minimum-dose protocols, the visibility of large structures in the axial plane was ranked as " 2 , clear" to " 3 , diminished" and only a slight but not significant improvement was perceived using VEO with respect to FBP (large structure mean scores 2.3 vs 2.5 ; repeated measures ANOVA, $P=0.05$, for all pair-wise comparisons) as shown in Fig. 4. Indeed, although VEO images have significantly less noise than those of FBP, VEO showed a blotchy pixilated appearance of the central bronchi and lung vessels (Fig. 1). On the other hand, for small structures scored from axial minimum-dose FBP images, the appearance of the anatomical details was insufficient for performing an adequate diagnostic and the structure visibility was rated as "diminished" to "non-diagnostic" with FBP, while the
Fig. 4 Radiologist scores of the five anatomical structures presented in Table 2 when visualized in the axial plane (four-point scale: 1 = excellent, 4 = non-diagnostic). For minimum-dose protocols, the structure visibility of the small structure group (periphery bronchi wall thickness, subpleural vessels and lung fissures) with VEO is higher than with FBP $(P<0.001)$ while the group of large structures (central bronchi and lung vessels) appears to be not significantly different $(P=0.05)$

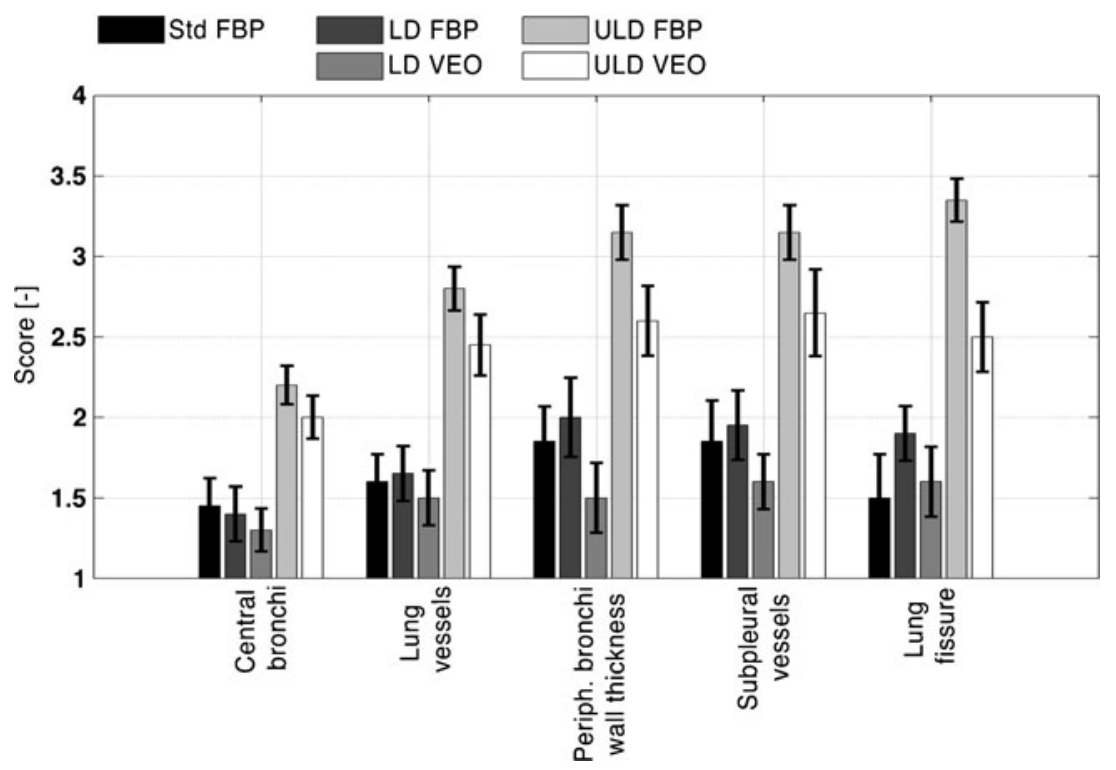


Fig. 5 Radiologist scores of the anatomical structures presented in Table 2 when visualized in the coronal plane (four-point scale: 1 = excellent, 4 = non-diagnostic). For minimum-dose protocols, the structure visibility of both structure groups is higher with VEO than with FBP (small structure group, $p<0.001$ and large structure group, $P<0.05$ )

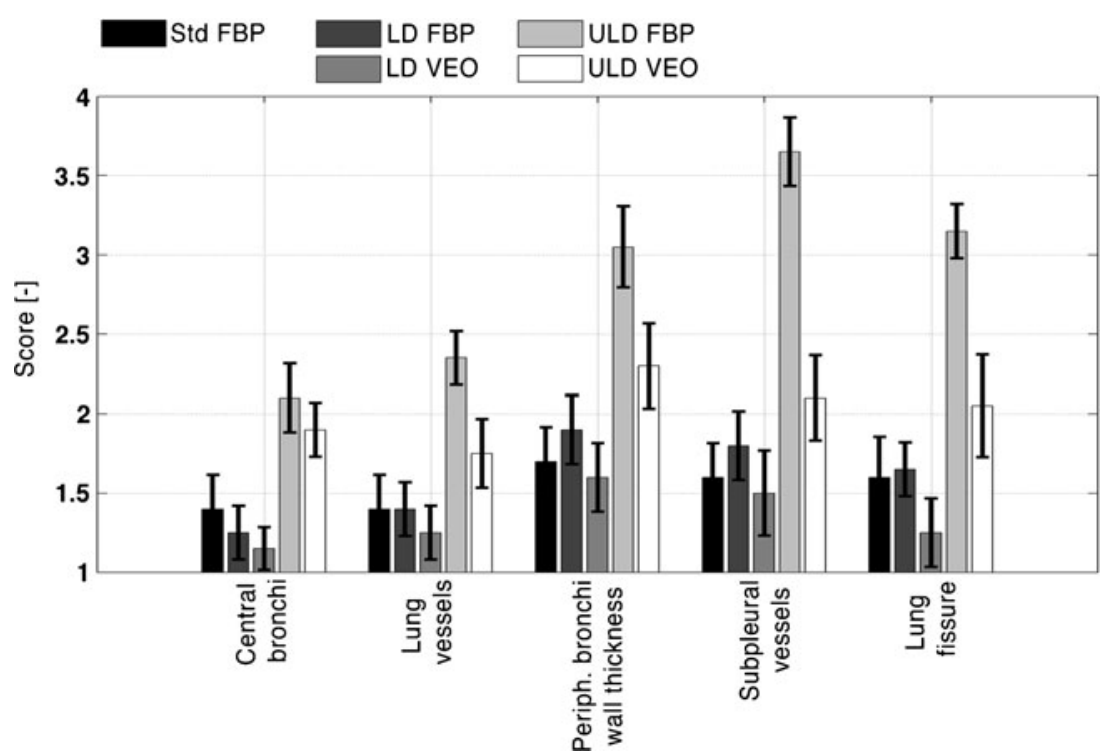

visibility of axial VEO images was ranked as "clear" to "diminished" (score 2.6 vs 3.3). This result corresponds to a significant improvement of the structure visibility with VEO (repeated measures ANOVA, $P<0.001$, for all pairwise comparisons).

The highest visibility enhancement was obtained for coronal images reconstructed with minimum-dose VEO and the ones reconstructed with minimum-dose FBP. While the visibility of large structures was slightly but significantly improved (large structure mean scores 1.9 vs 2.3; repeated measures ANOVA, $P<0.05$, for all pair-wise comparisons) and was scored as "clear" with both reconstructions, an important increase in visibility was observed for the small structures. Subpleural vessels and lung fissures, which were not detectable in certain cases on minimum-dose FBP images and were graded as "non-diagnostic", were ranked as "clear" to "diminished" visible (subpleural vessels mean scores 2.1 vs 3.7 ; lung fissures mean scores 2.1 vs 3.2; repeated measures ANOVA, $P<0.001$, for all pair-wise comparisons) as shown in Fig. 6. Although the differences in small structure visibility between moderately reduceddose FBP and minimum-dose FBP were important, differences between moderately reduced-dose VEO and minimum-dose VEO were strongly reduced (Fig. 5).
Fig. 6 At low dose and ultralow dose, the visibility of small structures (subpleural vessels and lung fissures) was strongly enhanced with VEO in comparison to FBP. With this latter reconstruction, some structures were even not discernable. a Coronal chest CT in an 11-yearold girl included in group A (standard protocol: CTDIvol $=$ 1.36 mGy) (b-e) Coronal chest $\mathrm{CT}$ in a 12-year-old boy included in group B (reduceddose protocol: CTDIvol $=$ $0.43 \mathrm{mGy}$; ULD protocol: CTDIvol $=0.14 \mathrm{mGy}$ )

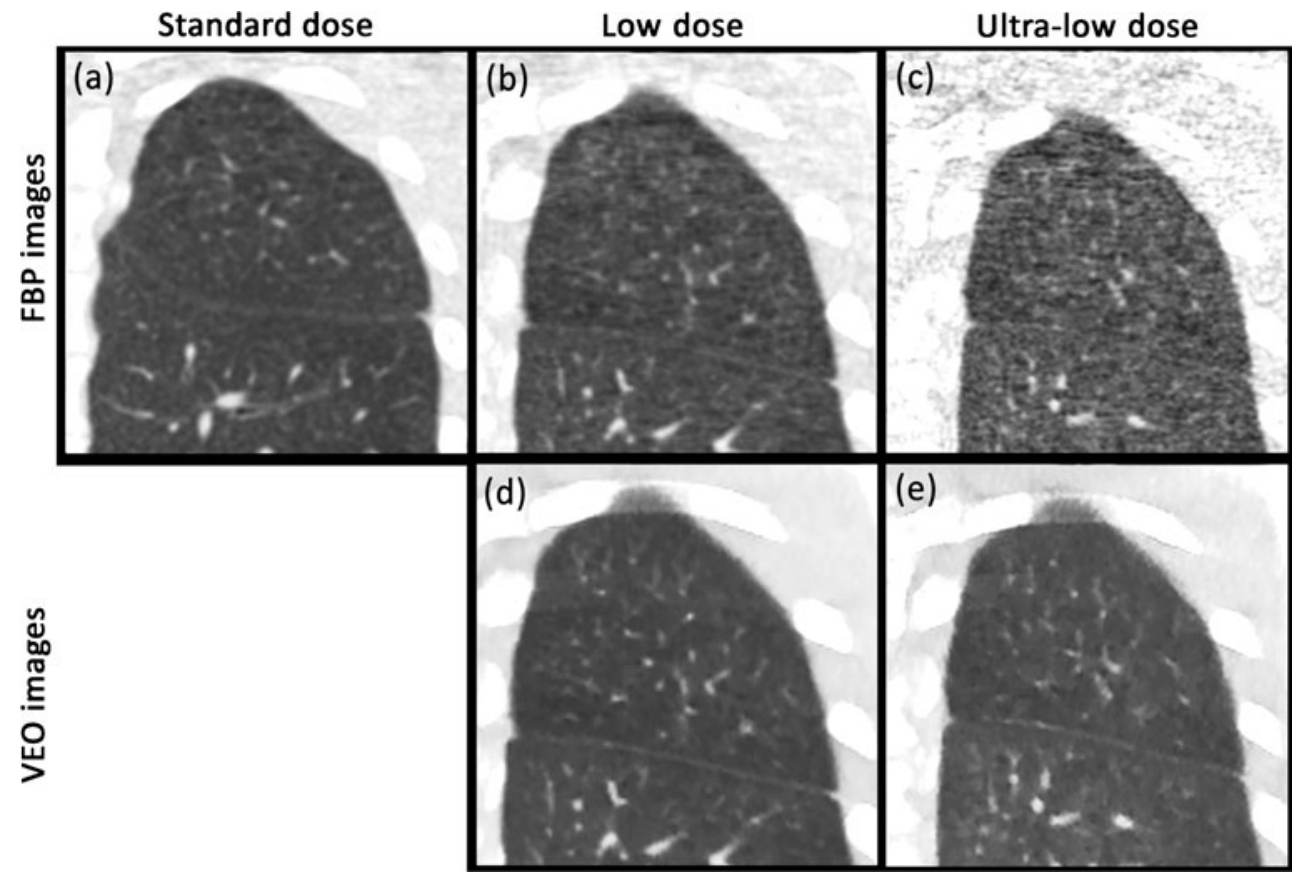




\section{Discussion}

The goal of this preliminary study was to evaluate the potential of VEO to reduce the radiation dose of pediatric chest $\mathrm{CT}$. By reconstructing the images with VEO and FBP, we found that the visibility of small anatomical structures was significantly improved with VEO in comparison with FBP. The greatest benefit was obtained for the subpleural vessels and lung fissures in the coronal plane, for which the visibility ranked from "non-diagnostic" or "diminished" with FBP to "clear" with VEO at minimum dose (Fig. 5). Although these structures were scored as "excellent" or "clear" when reconstructed with FBP at standard dose, our results showed that the potential for dose reduction could be very high (up to $92 \%$ ) depending on the clinical objective. With such a dose reduction, chest CT performed at minimum dose with VEO can reach dose levels close to those of posteroanterior chest radiography.

Various methods have been developed to reduce patient radiation dose, including automatic current modulation, weight-based tube voltage reduction, high pitch values on dual-source CT and, more recently, statistical iterative reconstructions [8-20]. In this preliminary study, we evaluated the first commercially available model-based iterative reconstruction, $\mathrm{VEO}^{\mathrm{TM}}$, as an effective tool to reduce dose in pediatric chest CT. Unlike the first iterative reconstructions, VEO requires a considerable amount of processing power and might not be used for all CT applications because of its long reconstruction time. In our study, it took 30$60 \mathrm{~min}$, depending on patient size and display field of view, to reconstruct one patient data set.

The main difficulty in clinical studies performed on children is to accurately evaluate image quality before determining a potential for dose reduction. In our study, we analyzed image quality using objective measurements and a subjective (or diagnostic) approach. In addition to CT numbers, SD and SNR, which were measured in patient images, two senior radiologists independently assessed the diagnostic quality of exams by using a four-point rating scale and a set of six criteria related to a chest CT examination (Table 2). By comparing various images of the same anatomical part of the body, this kind of approach could estimate the diagnostic image quality of patient examinations in a systematic and scientific manner [32].

No statistical differences in structure visibility were observed with FBP between children scanned at standard dose and those scanned at moderately reduced dose. This result shows that NI of our standard FBP protocol could have been increased in order to reduce dose, prior to this study, without significantly decreasing diagnostic image quality. By comparing objective and subjective noise, an important difference in SD was visible while overall subjective image quality remains around "clear" visibility, a 2 on the 4-point scale (Fig. 3). This confirms that the link between scalar measurements and radiologist perception is complex and that diagnostic image quality evaluated from anatomical criteria should always be considered as the reference.

The preliminary study revealed that VEO has a significantly higher potential to reduce the dose necessary for small structures compared to larger structures and this potential depends on the reconstruction plane. The clear improvement of the visibility of small structures in the coronal plane when compared to the axial plane is difficult to explain because FBP and VEO image sets were reformatted from the axial images in the same way. On the other hand, the modification of image texture produced by the VEO algorithm with respect to the FBP method could explain the difference between the visibility of small and large structures.

Interestingly, at minimum dose VEO is able to render visible certain small structures (subpleural vessels, lung fissures, etc.) that were not perceptible with FBP (Fig. 6). However, for larger structures already visible with FBP, the gain in visibility is less marked and dominated by the modification of image texture (Fig. 2). This change in image appearance is a new phenomenon that appeared with priorgeneration iterative reconstruction methods and has risen in recognition in the radiology community. With VEO at minimum dose, our two radiologists reported an alteration of the outlines of anatomical structures caused by a highlighting of the indented structure edge (Fig. 1). Nevertheless, in spite of the new image texture produced by VEO, no lower image conspicuity was reported for the set of normal chest structures investigated in this prospective study.

Our study has some limitations. The first was the relatively small number of patients due to the low frequency of $\mathrm{CF}$ examinations in our hospitals and the concern regarding CT radiation dose in this population. However, it is very likely that a study based on a large cohort would lead to similar results because of the relatively important number of structures already scored. The second limitation was the fact that we investigated a cohort of children ages 7-18 years with various morphotypes. This leads to more variability in the estimation of dose reduction with VEO, while the ACE system was used to reduce $\mathrm{CT}$ dose and fulfill as close as possible the as low as reasonably achievable (ALARA) requirements. Finally, it is important to emphasize that results were obtained for pediatric chest CT images visualized using the lung window settings. The benefit of VEO may differ significantly for different $\mathrm{CT}$ examinations such as abdomen scans when images are evaluated using standard window settings.

\section{Conclusion}

VEO significantly improved the visibility of small anatomical structures, such as subpleural vessels and lung fissures, 
visualized in coronal images with respect to the conventional FBP method.

Our preliminary results showed that the potential of VEO for dose reduction depends on the clinical objective, and that objective should be clearly defined prior to patient $\mathrm{CT}$ examinations. For small structures and cases involving pediatric follow-up, very high dose reduction (exceeding 90\%) might be achievable with VEO, although a long reconstruction time is still required.

Acknowledgments We would like to thank Professor Olivier Hélénon, chairman of the adult radiology department in the Necker Children's Hospital of Paris, for his assistance and for providing the machine time required to perform this study. This research was supported by the Swiss National Science Foundation grant [\#320030-120382].

\section{References}

1. Brenner DJ, Hall EJ (2007) Computed tomography: an increasing source of radiation exposure. N Engl J Med 357:2277-2284

2. Berrington de González A, Mahesh M, Kim KP et al (2009) Projected cancer risks from computed tomography scans performed in the United States in 2007. Arch Intern Med 169:2071-2077

3. Pierce DA, Shimizu Y, Preston DL et al (1996) Studies of the mortality of atomic bomb survivors. Report 12, part 1. Cancer: 1950-1990. Radiat Res 146:1-27

4. Brenner D, Elliston C, Hall E et al (2001) Estimated risks of radiation-induced fatal cancer from pediatric CT. Am J Roentgenol 176:289-296

5. McCollough CH, Bruesewitz MR, Kolfer JM (2006) CT dose reduction and dose management tools: overview of available options. Radiographics 26:503-512

6. Coursey C, Frush DP, Yoshizumi T et al (2008) Pediatric chest MDCT using tube current modulation: effect on radiation dose with breast shielding. Am J Roentgenol 190:W54-W61

7. Gunn MLD, Kohr JR (2009) State of the art: technologies for computed tomography dose reduction. Emerg Radiol 17:209-218

8. Silva AC, Lawder HJ, Hara A et al (2010) Innovations in CT dose reduction strategy: application of the adaptive statistical iterative reconstruction algorithm. Am J Roentgenol 194:191-199

9. Hara AK, Paden RG, Silva AC et al (2009) Iterative reconstruction technique for reducing body radiation dose at CT: feasibility study. Am J Roentgenol 193:764-771

10. Marin D, Nelson RC, Schindera ST et al (2010) Low-tube-voltage, high-tube-current multidetector abdominal CT: improved image quality and decreased radiation dose with adaptive statistical iterative reconstruction algorithm - initial clinical experience. Radiology 254:145-153

11. Leipsic J, Labounty TM, Heilbron B et al (2010) Adaptive statistical iterative reconstruction: assessment of image noise and image quality in coronary CT angiography. Am J Roentgenol 195:649-654

12. Leipsic J, Nguyen G, Brown J et al (2010) A prospective evaluation of dose reduction and image quality in chest CT using adaptive statistical iterative reconstruction. Am J Roentgenol 195:1095-1099

13. Prakash P, Kalra MK, Digumarthy SR et al (2010) Radiation dose reduction with chest computed tomography using adaptive statistical iterative reconstruction technique: initial experience. J Comput Assist Tomogr 34:40-45
14. Flicek KT, Hara AK, Silva AC et al (2010) Reducing the radiation dose for CT colonography using adaptive statistical iterative reconstruction: a pilot study. Am J Roentgenol 195:126-131

15. Sagara Y, Hara AK, Pavlicek W et al (2010) Abdominal CT: comparison of low-dose CT with adaptive statistical iterative reconstruction and routine-dose CT with filtered back projection in 53 patients. Am J Roentgenol 195:713-719

16. Singh S, Kalra MK, Hsieh J et al (2010) Abdominal CT: comparison of adaptive statistical iterative and filtered back projection reconstruction techniques. Radiology 257:373-383

17. Singh S, Kalra MK, Gilman MD et al (2011) Adaptive statistical iterative reconstruction technique for radiation dose reduction in chest CT: a pilot study. Radiology 259:565-573

18. Hu XH, Ding XF, Wu RZ et al (2011) Radiation dose of nonenhanced chest CT can be reduced $40 \%$ by using iterative reconstruction in image space. Clin Radiol 66:1023-1029

19. Pontana F, Pagniez J, Flohr T et al (2011) Chest computed tomography using iterative reconstruction vs. filtered back projection (part 1): evaluation of image noise reduction in 32 patients. Eur Radiol 21:627-635

20. Pontana F, Duhamel A, Pagniez J et al (2011) Chest computed tomography using iterative reconstruction vs filtered back projection (part 2): image quality of low-dose CT examinations in 80 patients. Eur Radiol 21:636-643

21. Miéville FA, Gudinchet F, Rizzo E et al (2011) Peadiatric cardiac CT examinations: impact of the iterative reconstruction method (ASIR) on image quality - preliminary findings. Pediatr Radiol 41:1154-1164

22. Thibault JB, Sauer KD, Bouman CA et al (2007) A threedimensional statistical approach to improved image quality for multislice helical CT. Med Phys 34:4526-4544

23. Miéville FA, Gudinchet F, Brunelle F et al (2012) Iterative reconstruction methods in two different MDCT scanners: physical metrics and 4-alternative forced-choice detectability experiments-a phantom approach. Phys Med. doi:10.1016/j.ejmp.2011.12.004 [Epub ahead of print]

24. Boone JM, Geraghty EM, Seibert JA et al (2003) Dose reduction in pediatric CT: a rational approach. Radiology 228:352-360

25. International Electrotechnical Committee (1994) Medical diagnostic x-ray equipment - radiation conditions for use in the determination of characteristics. Standard IEC \#61267, Geneva

26. International Electrotechnical Committee (1999) Medical diagnostic x-ray equipment - particular requirements for the safety of $\mathrm{x}$ ray equipment for CT. Standard IEC \#60601-2-44, Geneva

27. International Electrotechnical Committee (2002) Medical diagnostic $\mathrm{x}$-ray equipment - particular requirements for the safety of $\mathrm{x}$ ray equipment for CT, 2nd edition. Standard IEC \#60601-2-44, Geneva

28. BIPM, IEC, IFCC, ISO, IUPAC, IUPAP, OIML (2008) Evaluation of measurement data guide to the expression of uncertainty in measurement JCGM 100:2008 (GUM 1995 with minor corrections). BIPM Joint Committee for Guides in Metrology, Paris

29. Swiss Federal Office for Public Health (2010) Notice R-06-06. Bern, Switzerland. http://www.bag.admin.ch/themen/strahlung/ 02839/index.html?Lang=fr. Accessed 31 May 2010

30. Deak PD, Smal Y, Kalender WA (2010) Multisection CT protocols: sex- and age-specific conversion factors used to determine effective dose from dose-length product. Radiology 257:158-166

31. UNSCEAR (2000) UNSCEAR 2000: sources and effects of ionizing radiation. United Nations Scientific Committee on the Effects of Atomic Radiation, Vienna

32. Tingberg A, Hermann C, Lanhede B et al (2000) Comparison of two methods for evaluation of the image quality of lumbar spine radiographs. Radiat Prot Dosim 90:165-168 